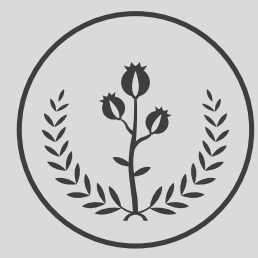

FUCS

\section{Re .

\title{
Perspectiva global y acción local para un programa doctoral en ciencias de la salud
}

\author{
Arley Gómez MD \\ Adriana Rojas MD \\ Omar Segura MD
}

\section{Global perspective and local actions for a doctoral program in health sciences}

${ }^{a}$ División de Investigaciones, Fundación Universitaria de Ciencias de la Salud, Bogotá DC, Colombia.

${ }^{b}$ Fundación Universitaria de Ciencias de la Salud, Bogotá DC, Colombia.

\section{R E S U M E N}

Con el objeto de ponderar las condiciones actuales requeridas para un programa doctoral en Ciencias de la Salud en Colombia, se elaboró un documento base y de posición para explorar su viabilidad y factibilidad de desarrollo. Mediante una estrategia de revisión teórica, discusión de expertos y definición de categorías, se establecieron temas centrales para el estudio y comprensión de la situación. A través de un proceso heurístico, se definieron cinco categorías clave; "historia de los doctorados", "perspectiva global", "situación nacional", "elementos en la FUCS" y "prospectiva", que muestran un panorama mundial de sobreoferta y precariedad laboral versus la necesidad y la posibilidad de generar localmente desarrollos e innovaciones en salud, a condición que un doctorado respete condiciones de excelencia, integralidad y protección del estudiante y del talento humano formado. La conclusión fundamental es que un programa PhD en Ciencias de la Salud es viable en la medida de la factibilidad y la fortaleza de factores tales como unos proyectos bien planteados y ejecutados, un equipo de supervisores y codirectores expertos y competentes, y un conjunto de capacidades administrativa, financiera y estructural, todo lo cual implica, al final, voluntades políticas y directivas.

Palabras clave: Ciencias de la salud; Colombia; educación de posgrado; política de educación superior.

(C) 2021 Fundación Universitaria de Ciencias de la Salud - FUCS. Este es un artículo Open Access bajo la licencia CC BY-NC-ND (http://creativecommons.org/licenses/by-nc-nd/4.0/).

\section{INFORMACIÓN DEL ARTÍCULO}

Historia del artículo:

Fecha recibido: septiembre 20 de 2019

Fecha aceptado: octubre 10 de 2019

\section{Autor para correspondencia. Dr. Omar Segura} odsegura@fucsalud.edu.co
DOI

10.31260/RepertMedCir.v29.n1.2020.974 
A base and position document was designed with the objective of considering the current conditions required to conceptualize a doctorate program in health sciences in Colombia, and explore its viability and feasibility. Some central topics were established by means of a theoretical review, expert discussion and defining categories in order to gain a better understanding of the situation. Five key categories were defined by an heuristic process, that is, "history of doctorates"; "global perspective"; "situation at the national level"; "elements available in the FUCS"; and "prospective", which show a global panorama of excess offer and job insecurity versus the need and the possibility to generate local health care developments and innovations to conduct a doctorate program respecting excellence, integrity and students and trained human talent. The fundamental conclusion was that a PhD program in health sciences depends on how feasible and strong some factors such as well formulated and implemented projects, a team of expert and competent supervisors and co-directors and a set of administrative, financial and structural capabilities are, all ultimately implying political and directive will.

Key words: health sciences; Colombia; postgraduate education; higher education policies.

(C) 2021 Fundación Universitaria de Ciencias de la Salud - FUCS. This is an open access article under the CC BY-NC-ND license ( ñhttp://creativecommons.org/licenses/by-nc-nd/4.0/).

\section{INTRODUCCIÓN}

El establecimiento de un programa doctoral en Ciencias de la Salud capaz de estimular la formación de alto nivel, soportado en pilares de una estructura filosófica-científica sólida y que aproveche la integración transdisciplinar y multidisciplinar, puede permitir el desarrollo de nuevo conocimiento a partir de la generación de lineamientos pedagógicos y la ejecución de proyectos de investigación básica y aplicada en el ramo. Esto, a su vez, puede constituir una fuente de innovaciones en la atención en salud, la solución de problemas clínicos y de salud pública, redundando en beneficios para la salud individual y colectiva. ${ }^{1}$

$\mathrm{Al}$ apreciarse una falta de integración entre las necesidades en salud de la sociedad colombiana, el desempeño y las capacidades de acción gubernamentales/estatales, y la oferta de la académica, se percibe la necesidad de contar con programas de alto nivel de formación, como lo son los doctorados, y al mismo tiempo, se evidencia la necesidad de poner en consonancia las tendencias mundiales académicas, sociales, societales y laborales de los futuros graduados de un programa doctoral junto a los parámetros dictados por el Ministerio de Educación Nacional (MEN) y el Consejo Nacional de Acreditación (CNA). Por consiguiente, el grupo ha operado y desarrollado este manuscrito alrededor de una pregunta central: ¿Cuáles son las perspectivas globales y las posibles acciones locales para desarrollar un doctorado en Ciencias de la Salud en la Fundación Universitaria de Ciencias de la Salud (FUCS)?

Por consiguiente, el objetivo fue explorar el panorama mundial de la formación doctoral en salud y ejecutar una prospección local de capacidades disponibles y elementos conceptuales y estructurales para ser desarrollados.

\section{MÉTODOS}

Se realizó una revisión teórica y exploración de contenidos a partir de tres grupos de fuentes de información: a) acervo documental educativo de la FUCS; b) manuscritos procedentes de repositorios como PubMed, Embase, Scielo, Redalyc, BVS, JSTOR, en formato HTML o PDF, en inglés o español, con las palabras de búsqueda "futuro", "doctorado", "PhD", "ciencias de la salud", en el período 2010-2018; y c) experiencias y vivencias de docentes en la Fundación Universitaria de Ciencias de la Salud (FUCS) con doctorado nacional o internacional o con desarrollos en investigación, con una experiencia mínima de 15 años/persona. Estos documentos fueron ingresados a una matriz de gestión de referencias usando el software Mendeley y compartidos para estudio vía Dropbox; el método está condensado iconográficamente en la (figura 1).

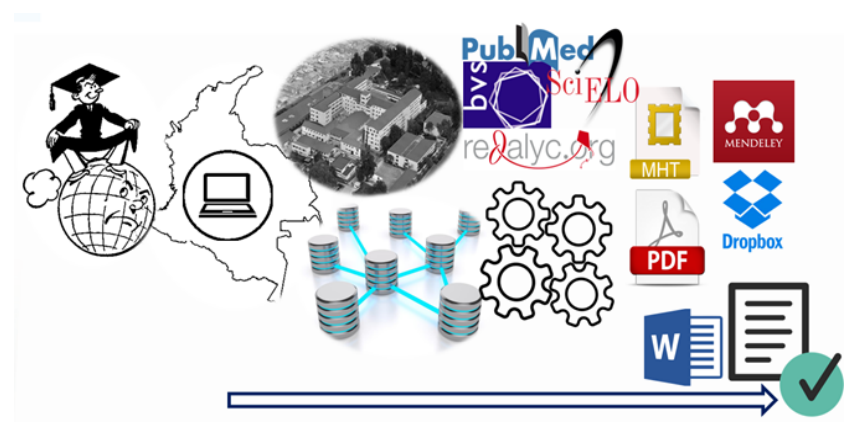

Figura 1 . Esquema del método empleado en el presente estudio. Fuente: los autores.

El proceso llevó a la generación de un documento posicional de estructura heurística (esto es, ajustable a partir de nuevos datos y comprensiones) basado en una estructura simple 
pasado-presente-futuro, centrado en cinco interrogantes convertidos en categorías: 1) historia de los doctorados a nivel mundial, regional o nacional, surgimiento y desarrollo; 2) perspectiva global, viabilidad y factibilidad de un doctorado; 3) situación de los doctorados en Colombia; 4) elementos disponibles o faltantes en la FUCS para un programa doctoral; y 5) prospectiva para un doctorado en el siglo XXI en Colombia.

\section{RESULTADOS}

Los resultados están condensados en la tabla 1. En la categoría "historia de los doctorados" la mayoría de los programas doctorales se ajustan a un modelo definido en las universidades europeas de la alta edad media., ${ }^{2,3}$ En esa época, los doctorados eran muy diferentes de los grados actuales porque era exigido un trabajo continuado sobre un área y no una investigación original sobre un tema, no había disertación ni tesis, sino un largo tiempo de residencia y aprendizaje tutelado, acompañado de exámenes periódicos. Más aún, en esas épocas tener maestría y doctorado era lo mismo que ser médico, teólogo o jurisconsulto y ser doctor era lo mismo. Sin embargo, maestría o doctorado no daban automáticamente derecho a enseñar, sino que la correspondiente licencia debía ser dada por la respectiva diócesis; esto cambió con la noción de "libertad de cátedra" presente en las constituciones democráticas modernas. Ello ha conllevado a alertas como las proferidas por Taylor ${ }^{3} \mathrm{y}$ Cyranoski ${ }^{4}$ sobre el colapso del mercado laboral académico en los países desarrollados, sin correcciones en las políticas de admisión o en los sobrecostos de la educación, teniendo como resultado estudiantes graduados para trabajar como asistentes laboratoriales y de enseñanza.
En la categoría "perspectiva global", Taylor ${ }^{3}$ planteó que el sistema doctoral mundial está desfinanciado, quebrado, es insostenible y necesita ser repensado; además, debe considerarse que el sistema se mueve no por la demanda del mercado laboral, sino por el aporte de fondos para investigación (grants) cuyo producto corresponde a publicaciones (papers) producidos por estudiantes $\mathrm{PhD}$ cada vez más precarios $\mathrm{y}$, también, más endeudados y enfermos. ${ }^{5}$ Se encuentra una influencia de la "economía naranja", de estímulo a la creatividad en el marco de un esquema económico de utilidad y rentabilidad mediado por la capacidad de generar y utilizar conocimiento. ${ }^{6,7}$

En la categoría situación nacional, el Consejo Nacional de Acreditación (CNA) ${ }^{2}$ ha advertido de un rezago en la formación a nivel doctoral en Colombia; los programas gradúan unos 150 doctores $\mathrm{PhD}$ por año en todas las áreas, dando por resultado una tasa de 4 a 6 doctores por millón de habitantes anual, tasa menor que la de otros países de Iberoamérica. Dada la importancia de los doctorados en el desarrollo científico, tecnológico y socioeconómico de cualquier país, hay ya en Colombia una política de fomento al desarrollo de los posgrados con especial énfasis en los doctorados, acelerando significativamente su crecimiento en los años venideros. Es así que mediante resolución 0036 de 2017 se plantea el apoyo para la formación doctoral por parte de Colciencias como ente rector de la investigación en Colombia, con la meta de llegar a formar 3.000 nuevos investigadores a nivel doctoral en el período 2018-2022. Esta situación conlleva a considerar aspectos tales como el perfil de salida, la sobreoferta, la proliferación o la disminución de calidad de la formación de los nuevos graduados $\mathrm{PhD}$, tal que los haga caer en situaciones de precariedad y de poca apreciación de la contribución de los $\mathrm{PhD}$ a la Academia, en particular la nacional; ${ }^{5,8-10}$ en el caso colombiano, se llevan a cabo - en medio de no pocas dificultades y obstáculos de

Tabla 1. Categorías emergentes y aspectos destacados

\begin{tabular}{|c|c|}
\hline Categoría & Destacado \\
\hline "Historia de los doctorados" & $\begin{array}{l}\text { - Modelo definido en las universidades europeas de la alta edad media. } \\
\text { - Trabajo continuado sobre un área y no una investigación original sobre un tema. } \\
\text { - Nihil obstat diocesano versus libertad de cátedra actual. } \\
\text { - Motivación de prestigio social versus financiera actual. }\end{array}$ \\
\hline "Perspectiva global” & $\begin{array}{l}\text { - Sistema actual doctoral es financieramente insostenible y debe cambiar. } \\
\text { - Sistema movido por fondos para investigación (grants), no por demanda laboral (1). } \\
\text { - Deterioro de condiciones de salud, económicas y laborales de los estudiantes PhD. }\end{array}$ \\
\hline "Situación nacional” & $\begin{array}{l}\text { - En Colombia se gradúan } 150 \text { doctores PhD/año (4-6/millón hab.) en todas las áreas. } \\
\text { - Desconocimiento de perfiles de egresados, sin integración con dinámicas nacionales. } \\
\text { - Alta incorporación de tecnologías foráneas sin evidencia ni adaptación suficientes. } \\
\text { - Necesidades de infra/superestructura para generar investigación e innovación. }\end{array}$ \\
\hline “Elementos en la FUCS” & $\begin{array}{l}\text { - Experiencia centenaria formando especialistas médico-quirúrgicos-científicos. } \\
\text { - Hospitales certificados como "universitarios de alta calidad", con investigación. } \\
\text { - Participación en redes (inter)nacionales de educación/investigación en salud. } \\
\text { - Gestión organizacional por procesos. }\end{array}$ \\
\hline “Perspectiva global” & $\begin{array}{l}\text { - Según Sørensen17 y Keller y col.18 un buen programa doctoral permite: } \\
0 \text { entrenar a los doctorandos en métodos de investigación y en interacción social. } \\
0 \text { ampliar el conocimiento en salud mediante proyectos de investigación. }\end{array}$ \\
\hline
\end{tabular}

Fuente: Ios autores. 
diversa índole, como nuevos descubrimientos científicos en áreas diagnósticas, terapéuticas, de prevención y seguimiento de las distintas enfermedades humanas. Sin embargo, en gran número de casos aún existe incorporación de tecnologías foráneas sobre las cuales falta evidencia suficiente de su eficacia en nuestra población.

En la categoría elementos en la FUCS, la institución tiene una experiencia mayor de 100 años en la formación de profesionales con posgrado médico-quirúrgico, en profesiones de la salud como medicina, enfermería, instrumentación quirúrgica, citohistología y más recientemente psicología y administración en salud; cuenta con hospitales certificados como "universitarios de alta calidad" y grupos y servicios médico-quirúrgicos con gran experiencia operativa y de trabajo en equipo; tiene investigación basada en hospitales, con programas educativos orientados a la formación de subespecialistas que bajo supervisión hacen en general investigación de índole formativa y en ocasiones aplicada, generando nuevo conocimiento; participa de redes nacionales e internacionales de educación e investigación en salud y ha demostrado capacidad para crear, implementar y evaluar nuevas teorías, ${ }^{11}$ técnicas y tecnologías, ${ }^{12}$ guías de práctica clínica, $^{13}$ protocolos clínicos ${ }^{14} \mathrm{y}$ otros procedimientos, de conformidad con las funciones sustantivas consignadas en su Proyecto Educativo Institucional (PEI) ${ }^{15}$ y su Plan Estratégico 2016-2019. ${ }^{16}$ A partir de esto, necesita proseguir y completar un proceso de inversión en infra/superestructura y desarrollo de capacidades operativas e innovativas.

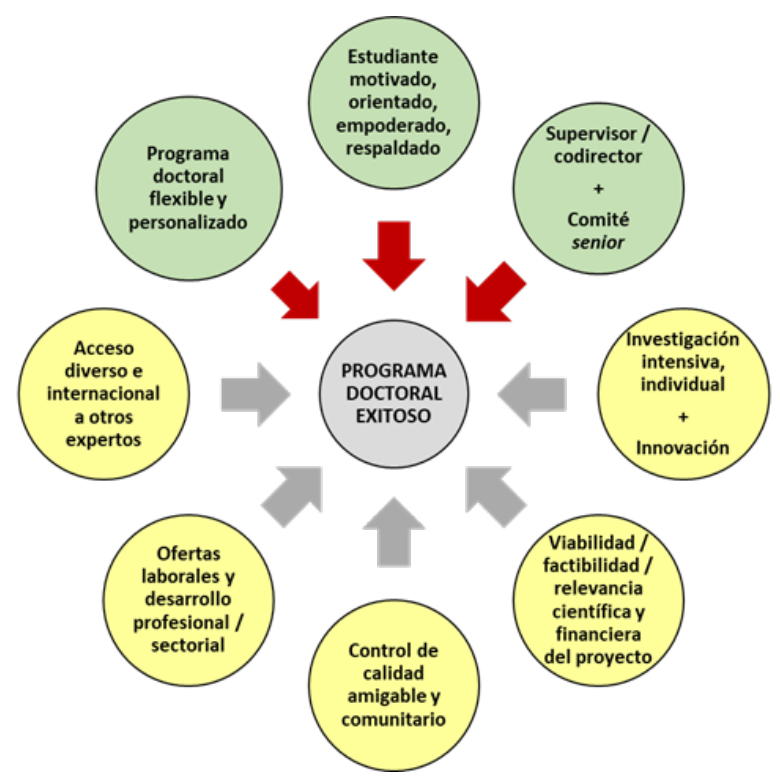

Figura 2. Elementos para un programa doctoral exitoso. Fuente: desarrollado a partir de Keller y col. ${ }^{18}$

En la categoría prospectiva, se encuentran diferentes documentos que apelan a la integridad, sea holismo o eclecticismo, en pos de un proceso satisfactorio para estudiantes, profesores, programas y sociedades. Para
Sørensen, ${ }^{17}$ un programa doctoral se orienta a dos objetivos fundamentales: ampliar el conocimiento acerca de un tópico de salud importante e inexplorado mediante un proyecto de investigación y entrenar extensiva e intensivamente a los estudiantes doctorales no sólo en métodos de investigación, sino también en interacción social. Keller y col. ${ }^{18}$ por su parte señalan ocho elementos para un programa exitoso: 1) ejecución de investigación en un medio que la soporte a un ritmo intensivo, con un carácter individual y original; 2) supervisión del estudiante doctoral por un comité senior o de excelencia; 3) existencia de estructuras programáticas flexibles para el desarrollo personal y profesional; 4) existencia de portafolio programático personalizado para cada estudiante doctoral; 5) diversidad e internacionalización; 6) garantías de fondos o de financiación para la duración de los doctorados; 7) oportunidades para un control de calidad amigable y mancomunado, y 8) ofertas laborales y de desarrollo profesional interdisciplinares o intersectoriales (figura 2).

\section{ISCUSIÓN}

El decreto 1295 de 2010, ${ }^{19}$ art. 25, define como programa de doctorado aquel que "tiene como propósito la formación de investigadores con capacidad de realizar y orientar en forma autónoma procesos académicos e investigativos en un área especifica del conocimiento y desarrollar, afianzar o profundizar competencias propias de este nivel de formación. Los resultados de las investigaciones de los estudiantes en este nivel de formación deben contribuir al avance en la ciencia, la tecnología, las humanidades o las artes".

El mismo decreto, en su capítulo II, establece una serie de condiciones para obtener el registro calificado de un programa. Brevemente, estos son: denominación, justificación (estado del arte, necesidades nacionales y rasgos distintivos), contenidos curriculares (fundamentación teórica, propósitos de formación, plan general de estudios, interdisciplinariedad, flexibilización, estrategias pedagógicas, metodológicas y didácticas, currículo, pensum, syllabi), organización de actividades académicas (para personal de salud, involucra los modelos de docencia-servicio contemplados en la ley 1164 de 2007), investigación (promoción, procedimientos tecnológicos, medio ambiente investigativo, procesos, grupos, productos), personal docente (estructura organizacional, núcleo, trayectoria, experiencia, idoneidad, disponibilidad, vinculación, educación continua, estatuto), relación con sector externo (nexos o vinculaciones, trabajo con comunidad y proyección social, impactos y desempeños laborales, prospección de potenciales egresados, generación de nuevo conocimiento), medios educativos, infraestructura física.

La Fundación Universitaria de Ciencias de la Salud- FUCS, los hospitales de San José e Infantil Universitario de San José y la Sociedad de Cirugía de Bogotá (SCB), están comprometidos en el apoyo a la investigación científica aplicada, la formación de excelencia y el desarrollo de protocolos clínicos, así como en el diseño e implementación de políticas en salud orientadas 
a población vulnerable a nivel local, regional y nacional, de conformidad con las funciones sustantivas consignadas en su Proyecto Educativo Institucional (PEI) ${ }^{15}$ y su Plan Estratégico 2016-2019. ${ }^{16}$ Del presente ejercicio de revisión teórica, la conclusión fundamental es que el Doctorado es viable en la medida de la fortaleza de factores tales como unos proyectos bien planteados y ejecutados, un equipo de supervisores y codirectores expertos y competentes, y el desarrollo continuado y sostenido a largo plazo del conjunto de

\section{REFEREN CI A S}

1. Fundación Universitaria de Ciencias de la Salud. Documento maestro. Doctorado en Ciencias de la Salud. Bogotá: Fundación Universitaria de Ciencias de la Salud; 2017. 35 p.

2. Ministerio de Educación Nacional, Consejo Nacional de Acreditación. Lineamientos para la Calidad de programas de Maestría y doctorados. 2010. 1-34 p.

3. Taylor M. Reform the PhD system or close it down. Nature. 2011;472(7343):261.

4. Cyranoski D, Gilbert N, Ledford H, Nayar A, Yahia M. Education: The PhD factory. Nature2. 2011;472(7343):276-9.

5. Fiske $\mathrm{P}$. What is a $\mathrm{PhD}$ really worth? Nature [Internet]. 2011;472(7343):381. Disponible en: http://www.nature.com/ doifinder/10.1038/nj7343-381a

6. Buitrago F, Duque Márquez I. La economía naranja. Una oportunidad infinita. Washington D.C.: Banco Interamericano de Desarrollo; 2013. 244 p.

7. Finlev T, Rachel M, Oppenheim B, Skvirsky S. El futuro de la economíanaranja [Internet]. WashingtonD.C.: BancoInteramericano de Desarrollo; 2016 [citado 2019 febrero]; Recuperado de: https://publications.iadb.org/bitstream/handle/11319/8459/ VersionFinalPortugues.PDF? sequence $=9$ \&isAllowed $=\mathrm{y}$

8. Anzola Montero G. Realidad de los Posgrados en Colombia y su situación frente a la reforma de la Ley 30. Rev UDCA [Internet]. 2011;14(1):3-5. Disponible en: http://www.scielo.org.co/pdf/ rudca/v14n2/v14n2a01.pdf

9. Segura O. Economía de la salud y salud pública: Situación global y perspectivas locales. Biomédica. 2018;38(2).

10. Ardila A. Neoliberalismo y trabajo médico en el Sistema General de Seguridad Social en Salud [Internet]. 2016 [citado 2019 febrero]; Recuperado de: http://www.bdigital.unal.edu. co/53386/1/598690.2016.pdf\%5Cnhttp://www.bdigital.unal.edu. $\mathrm{co} / 53386 /$

11. Segura O, Duque MG, Enciso C, Porras OC. Agotamiento profesional (burnout) en médicos intensivistas: una visión de la unidad de cuidados intensivos desde la teoría fundamentada. capacidades administrativa, financiera y estructural al interior de la Fundación.

\section{CONFLICTO DE INTERESES}

Los coautores de este manuscrito declaramos no tener conflicto de intereses.

Acta Colomb Cuid Intensivo [Internet]. 2016;16(3):126-35. [citado 2019 febrero]; Recuperado de: www.elsevier.es/accihttp://dx.doi. org/10.1016/j.acci.2016.04.0030122-7262/

12. Prada-Madrid JR, Franco-Chaparro LP, Garcia-Wenninger M, Palomino-Consuegra T, Stanford N, Castaneda-Hernandez DA. A Surgical Technique for Management of the Metopic Suture in Syndromic Craniosynostosis. J Craniofac Surg. 2017;28(3):675-8.

13. Acosta A, Nova J, Sánchez G, Rodríguez A, Rueda X. Guía de atención integral con evaluación económica para la prevención, el diagnóstico, el tratamiento y el seguimiento del cáncer de piel no melanoma: carcinoma basocelular. 2015;4:258-96.

14. Muñoz E, Mejía S, Correa JR. Signo del Hospital de San José, una alternativa sencilla para el pivot shift para diagnóstico de inestabilidad anterolateral de la rodilla por lesión del ligamento cruzado anterior. Rev Colomb Ortop Traumatol. 2014;28(1):13-5.

15. FUCS. Proyecto Educativo Institucional [Internet]. Fundación Universitaria de Ciencias de la Salud, editor. Bogotá; 2014. 69 p. Disponible en: http://www.fucsalud.edu.co/themes/custom/fucs/ images/documentos-FUCS/PEI_FUCS.pdf

16. FUCS. Plan Estratégico de Desarrollo 2016-2019 [Internet]. Fundación Universitaria de Ciencias de la Salud, editor. Bogotá; 2015. 107 p. [citado 2019 febrero]; Recuperado de: http://www. fucsalud.edu.co/themes/custom/fucs/images/documentos-FUCS/ PED-2016-2019.pdf

17. Sørensen HT. I-determinants for a successful $\mathrm{PhD}$ or postdoctoral outcome. Clin Epidemiol. 2016;8:297-303.

18. Keller F, Dhaini S, Briel M, Henrichs S, Höchsmann C, Kalbermatten $\mathrm{D}$, et al. How to Conceptualize and Implement a PhD Program in Health Sciences-The Basel Approach. J Med Educ Curric Dev [Internet]. 2018;5:238212051877136. Disponible en: http:// journals.sagepub.com/doi/10.1177/2382120518771364

19. Presidencia de la República de Colombia. Decreto por el cual se reglamenta el registro calificado de que trata la Ley 1188 de 2008 y la oferta y desarrollo de programas académicos de educación superior. Decreto 1295 de 2010 (20 de abril). 